\title{
STEMI Due to Big Ostial Left Main Thrombus Extending Into Aorta: Challenging Situation With No Clear Guidelines
}

\author{
Mohammady Shahin ${ }^{\mathrm{a}}$, Oliver Gaemperlia, Patrick Siegrist ${ }^{\mathrm{a}}$, \\ Jasmina Alibegovic ${ }^{\mathrm{a}, \mathrm{b}}$
}

\begin{abstract}
Extensive left main (LM) coronary artery thrombus is a rare and lifethreatening angiographic finding with usual dramatic clinical presentation including hemodynamic instability and sudden cardiac death. The proper management of a big LM thrombus extending into aorta remains a challenging issue with no clear guidelines. In the following case report we present a 45 -year-old patient with no apparent risk factors for coronary artery disease who presented with acute inferolateral ST-elevation myocardial infarction (STEMI). The examination was performed using a right transfemoral approach and it revealed the presence of a large mobile, hazy mass within the left main coronary artery (LMCA) extending into the aorta. To confirm the extension of that structure we performed intravascular ultrasound (IVUS) which revealed a circumferential mass in the LMCA extending with its twothirds into the aorta with no evidence of atherosclerotic plaques. After a long discussion within our Heart team we decided to transfer the patient for urgent surgical removal. Such decision was made with regard to the large size of the mass and in order to avoid systemic or distal embolization into coronary arteries. Perioperative transesophageal echocardiography (TEE) confirmed diagnosis and excluded presence of patent foramen ovale (PFO). Surgical removal was done successfully with complete resolution of ST-segment elevation and rapid fall of cardiac enzymes to normal levels. Postoperative course was uneventful. The mass was defined as a thrombus by pathophysiology examination. Patient was discharged well from our hospital after 1 week.
\end{abstract}

Keywords: Acute coronary syndromes; Left main disease; STEMI

\section{Introduction}

Extensive left main (LM) coronary artery thrombus is a rare and life-threatening angiographic finding with usual dramatic

Manuscript submitted July 10, 2017, accepted September 18, 2017

aUniversity Heart Center, Department of Cardiology, University Hospital Zurich, Zurich, Switzerland

${ }^{b}$ Corresponding Author: Jasmina Alibegovic, University Heart Center, University Hospital Zurich, Raemistrasse 100, CH-8091 Zurich, Switzerland.

Email: Jasmina.Alibegovic@usz.ch

doi: https://doi.org/10.14740/cr598e clinical presentation including hemodynamic instability and sudden cardiac death [1-3]. Fibrous cap rupture of an atherosclerotic plaque followed by thrombus formation is the most common mechanism while other factors including hypercoagulable states can be involved [2]. The proper management of a big LM thrombus extending into aorta remains a challenging issue with no clear guidelines. The following case report describes a successful surgical removal of the large LM main coronary artery thrombus in the setting of ST-segment elevation myocardial infarction.

\section{Case Report}

A 45-year-old patient with no apparent risk factors for coronary artery disease was referred from a peripheral hospital to our catheterization laboratory for a primary percutaneous coronary intervention (PCI). He presented with retrosternal burning chest pain, he was hemodynamically stable and the ECG showed acute infero-lateral ST-elevation myocardial infarction (Fig. 1). The examination was performed using a right transfemoral approach and it revealed the presence of a large mobile, hazy mass within the left main coronary artery (LMCA) extending into the aorta while left anterior descending (LAD), left circumflex (LCX) and the right coronary artery (RCA) appeared angiographically normal (Figs. 2-4) (Supplementary videos 1 and 2, www. cardiologyres.org). To confirm the extension of that structure we performed intravascular ultrasound (IVUS) which revealed a circumferential mass in the LMCA extending with its two-thirds into the aorta with no evidence of atherosclerotic plaques (Fig. 5). After a long discussion within our Heart team we decided to transfer the patient for urgent surgical removal. Such decision was made with regard to the large size of the mass and in order to avoid systemic or distal embolization into coronary arteries. Perioperative transesophageal echocardiography (TEE) equally showed a large pendulating mass in the LMCA expanding to the aorta (Fig. 6). Surgical removal was done successfully. The patient was transferred to the coronary intensive care unit hemodynamically stable. The ST elevation immediately subsided. Troponin T high sensitivity peak measurement was initially $911 \mathrm{ng} / \mathrm{L}$ (Table 1) and during the following days presented a declining course. Postoperative course was uneventful. The mass was defined as a thrombus by pathohistology examination (Fig. 7). Patient was discharged well from our hospital after 1 week admission to 


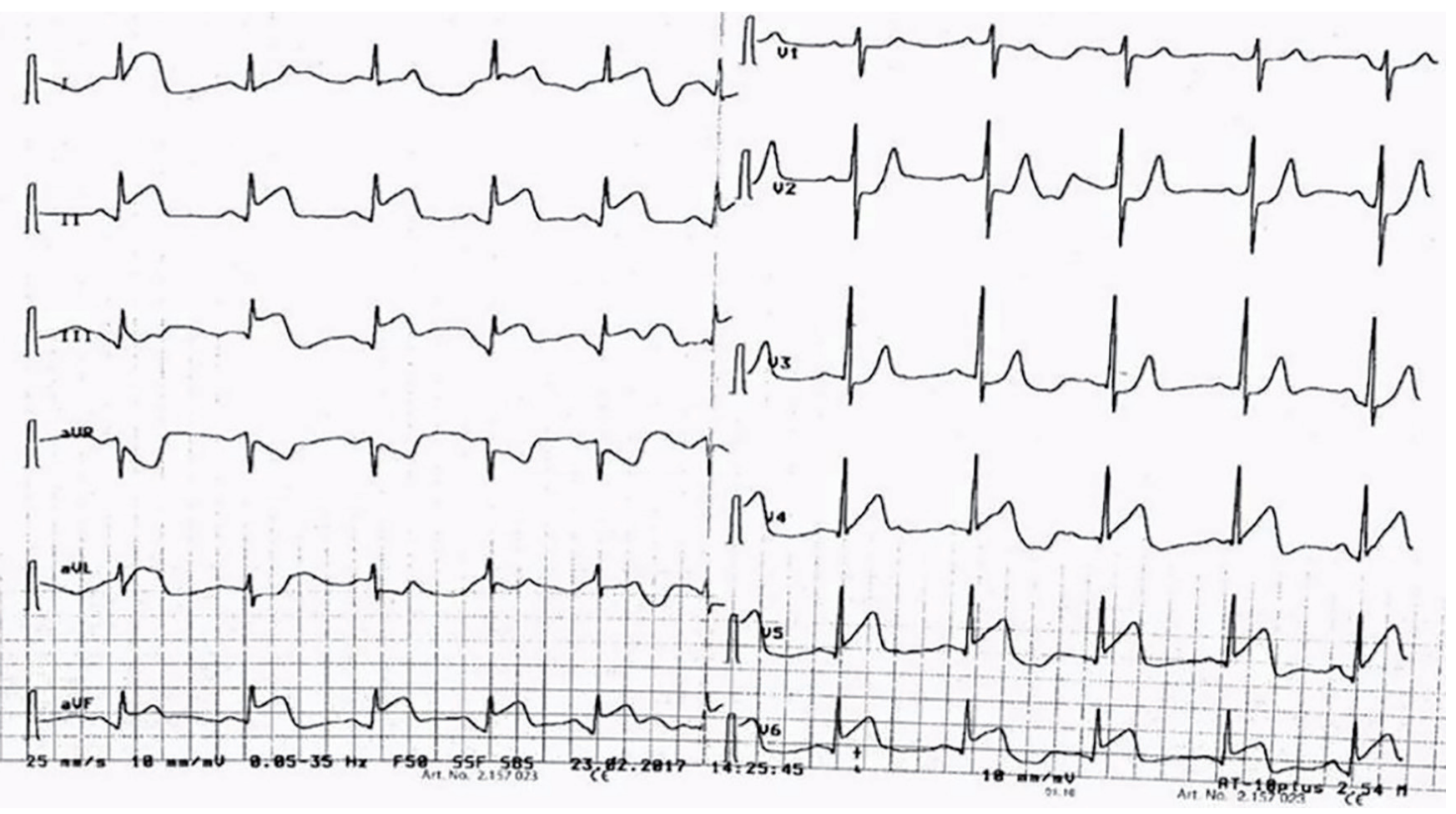

Figure 1. ECG showing STE in infero-lateral leads. ECG: electrocardiogram; STE: ST elevation.

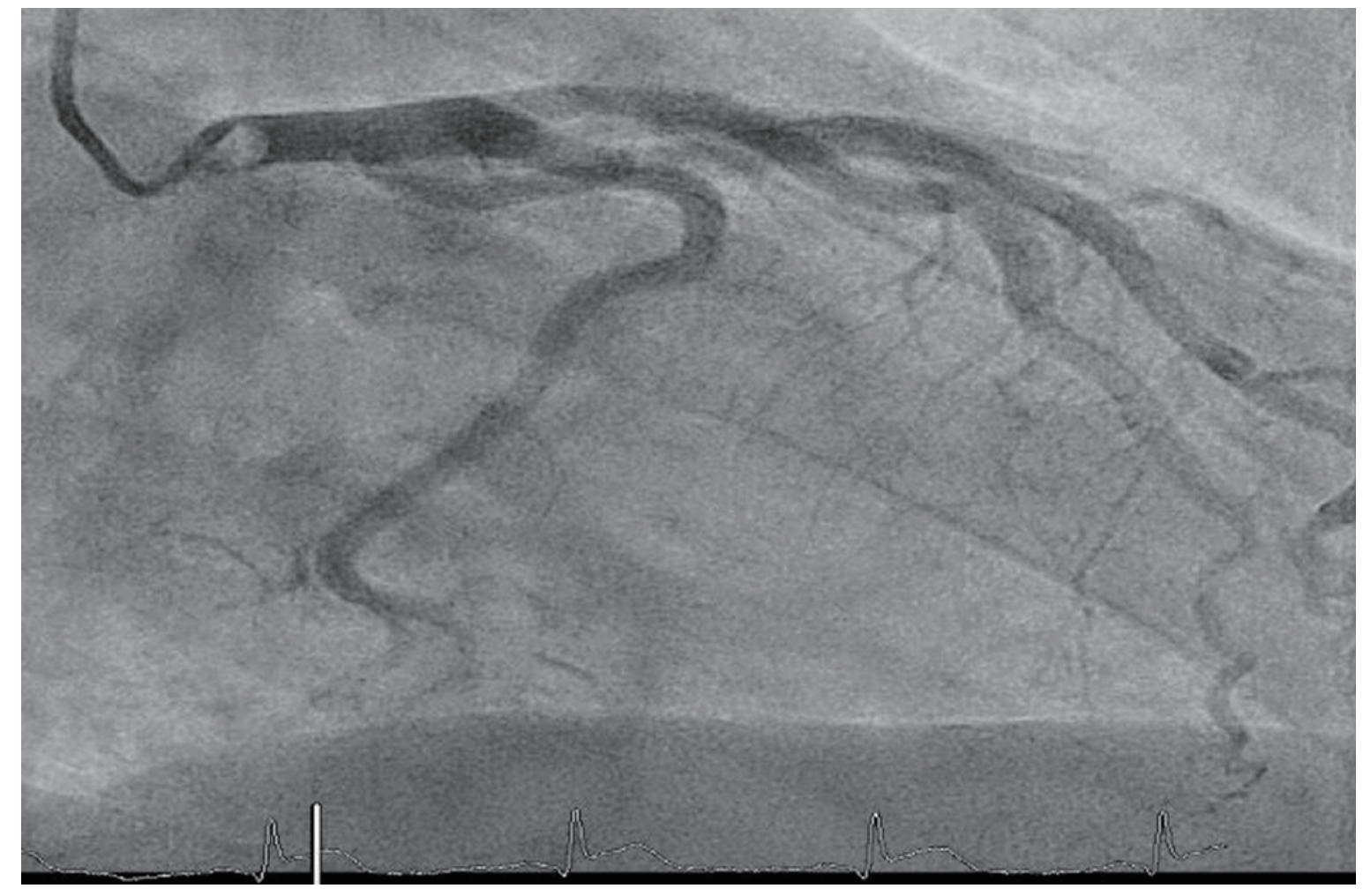

Figure 2. Coronary angiography RAO 8/Caudal 0 view showing big ostial LM thrombus with normal LAD, LCX and RIM arteries. RAO: right anterior oblique; LM: left main; LAD: left anterior descending; LCX: left circumflex; RIM: ramus intermedius artery. 


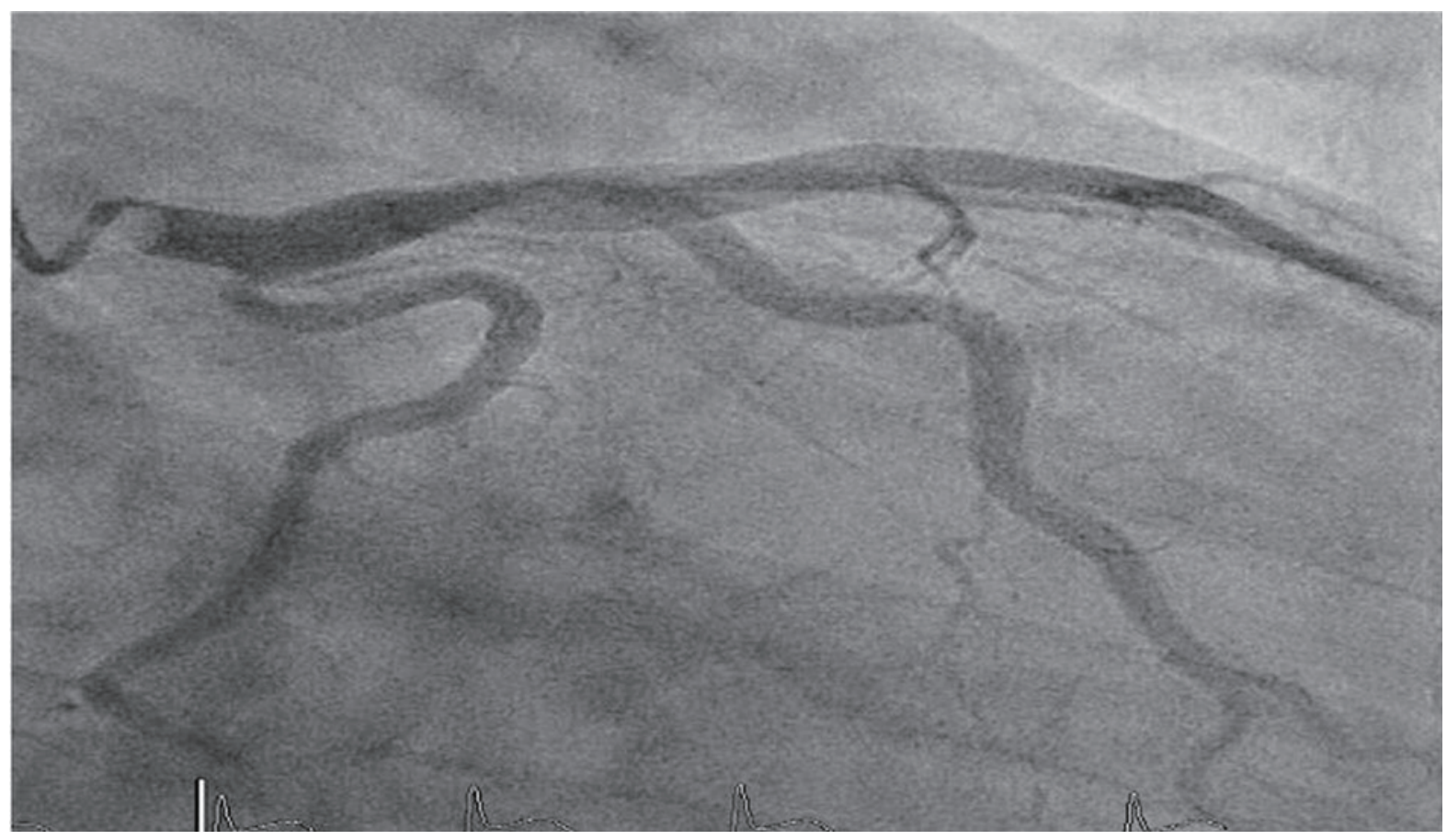

Figure 3. Coronary angiography RAO 30/Caudal 18 view showing big LM thrombus with normal LAD, LCX and RIM arteries. RAO: right anterior oblique; LM: left main; LAD: left anterior descending; LCX: left circumflex; RIM: ramus intermedius artery.

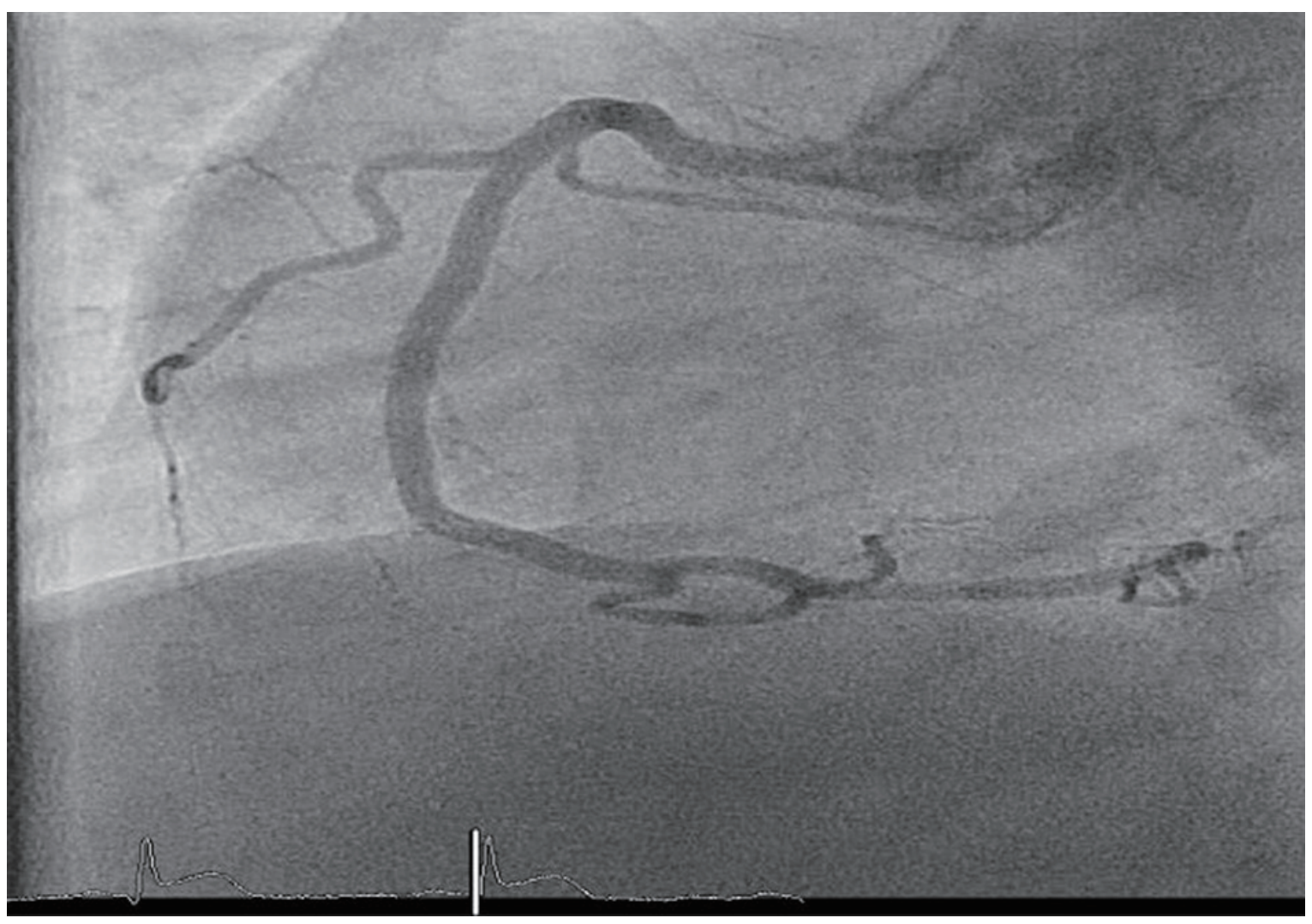

Figure 4. Coronary angiography LAO 52/Caudal 15 view showing normal RCA. LAO: left anterior oblique; RCA: right coronary artery. 


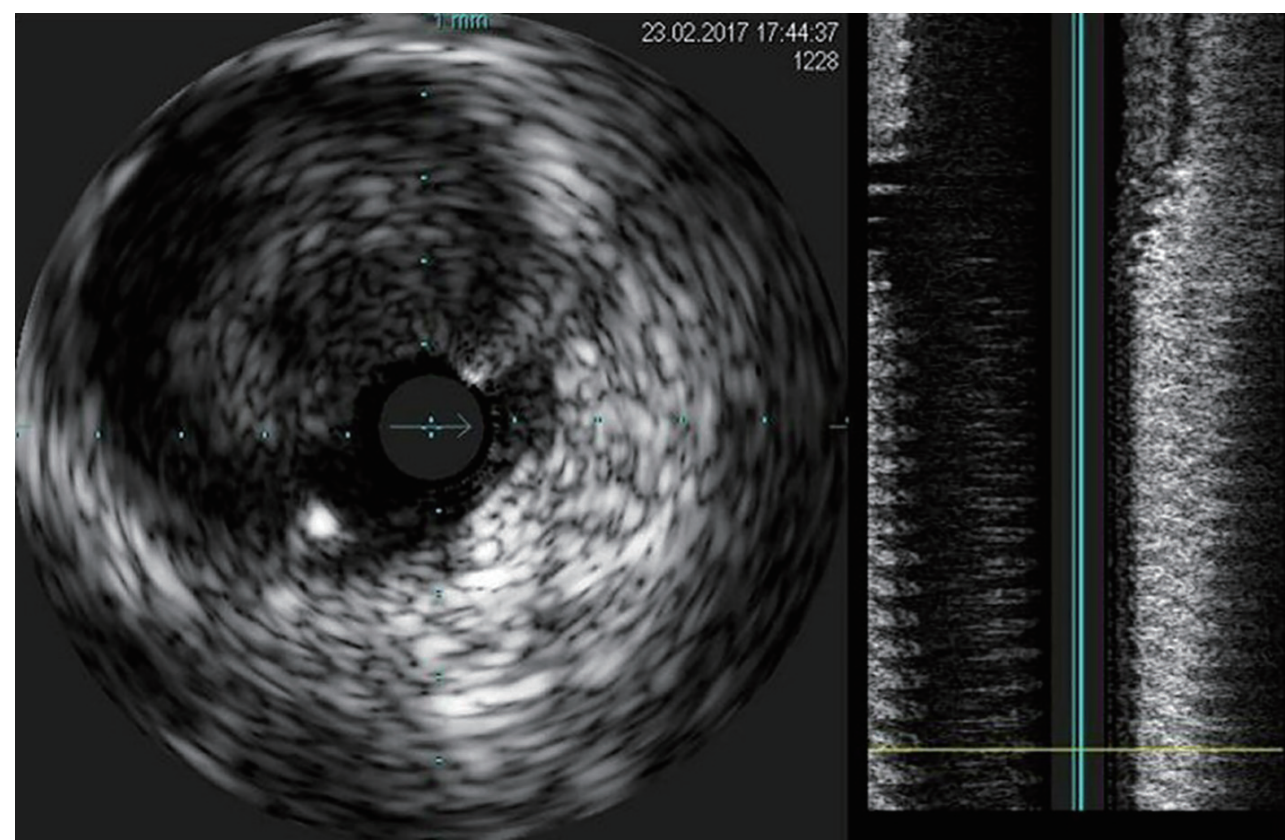

Figure 5. IVUS image showing big ostial LM thrombus. IVUS: intravascular ultrasound; LM: left main.

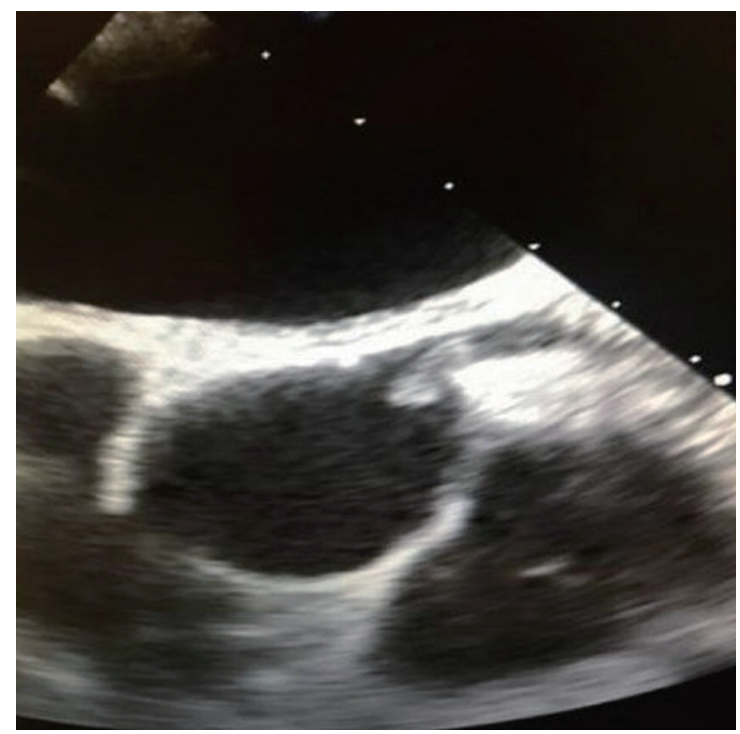

Figure 6. Intraoperative TEE showing big ostial LM thrombus extending into aorta. TEE: transesophageal echocardiography; LM: left main.

Table 1. Laboratory Tests at Presentation

\begin{tabular}{ll}
\hline Test & Results \\
\hline CK total $(<190 \mathrm{U} / \mathrm{L})$ & $920 \mathrm{U} / \mathrm{L}$ \\
Myoglobin $(28-72 \mu \mathrm{g} / \mathrm{L})$ & $600 \mu \mathrm{g} / \mathrm{L}$ \\
Troponin T high sensitivity $(<14 \mathrm{ng} / \mathrm{L})$ & $911 \mathrm{ng} / \mathrm{L}$ \\
NT-proBNP $(<85.8 \mathrm{ng} / \mathrm{L})$ & $18 \mathrm{ng} / \mathrm{L}$ \\
Creatinine $(44-80 \mu \mathrm{mol} / \mathrm{L})$ & $66 \mu \mathrm{mol} / \mathrm{L}$ \\
\hline
\end{tabular}

CK: creatine kinase; NT: N-terminal; BNP: brain natriuretic peptide.

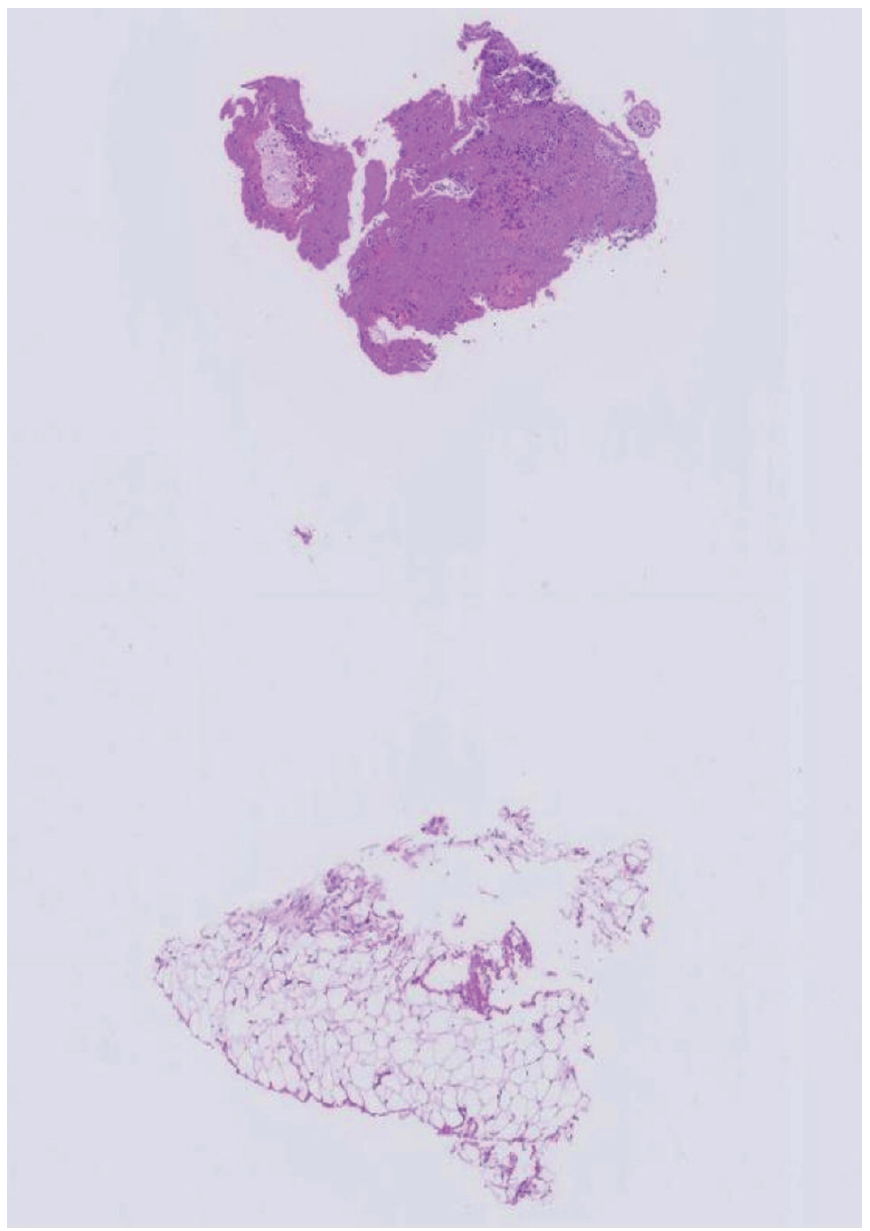

Figure 7. Histopathological picture from obtained thrombus material. 
another hospital near to his residence in another canton.

\section{Discussion}

Large size LMCA thrombus is a rare and life-threatening angiographic finding with usual dramatic clinical picture including hemodynamic instability and sudden cardiac death. The proper management in this situation remains a challenging issue with no clear guidelines $[4,5]$. In our case, we decided to refer the patient for urgent surgical removal in order to avoid thrombus disintegration and potential systemic or distal coronary embolization. Surgical removal was done successfully with complete resolution of ST-segment elevation and rapid fall of cardiac enzymes to normal levels.

\section{References}

1. Klein AJ, Casserly IP, Messenger JC. Acute left main coronary arterial thrombosis - a case series. J Invasive Cardiol. 2008;20:243-246.

2. Prasad SB, Whitbourn R, Malaiapan Y, Ahmar W, MacIsaac A, Meredith IT. Primary percutaneous coronary intervention for acute myocardial infarction caused by unprotected left main stem thrombosis. Catheter Cardiovasc Interv. 2009;73(3):301-307.

3. Patel M, Bhangoo M, Prasad A. Successful percutaneous treatment of suspected embolic left main thrombosis in a patient with a mechanical aortic valve. J Invasive Cardiol. 2011;23(11):E263-266.

4. De Luca G, Suryapranata H, Thomas K, van 't Hof AW, de Boer MJ, Hoorntje JC, Zijlstra F. Outcome in patients treated with primary angioplasty for acute myocardial infarction due to left main coronary artery occlusion. Am J Cardiol. 2003;91(2):235-238.

5. Maddoux GL, Goss JE, Ramo BW, Raff GL, Heuser RR, Shadoff N, Wilson JN, et al. Left main coronary artery embolism: a case report. Cathet Cardiovasc Diagn. 1987;13(6):394-397. 